\title{
柔性比较分子场分析方法在糖原磷酸化酶抑制剂 三维定量构效关系研究中的应用
}

\author{
周 鹏 李志良 ${ }^{*}$
}

(重庆大学化学化工学院, 重庆 400044)

\begin{abstract}
摘要通过取消比较分子场分析法(CoMFA)中作为探针安置的网格格点, 并辅以群体智能算 法来优化不同类型探针在药物分子周围最佳分布情况, 以此把柔性机制引入到 CoMFA 当中, 从 而形成了一种新的 3D-QSAR 研究方法: 柔性比较分子场分析法(FCoMFA). 初步尝试使用 FCoMFA 对 47 个糖原磷酸化酶抑制剂研究结果表明: FCoMFA 能够有效找寻配基与受体的活性 位点作用方式并给出具有较强稳定性和预测能力的 QSAR 模型, 同时可直观通过探针分布模式 图观察不同取代基对药物活性的影响情况.
\end{abstract}

\section{关键词柔性比较分子场分析法 三维定量构效关系 粒子群优化算法 糖原磷酸化酶抑制剂 探针分布模式图}

1988 年由Cramer等 ${ }^{[1]}$ 提出的比较分子场分析法 (COMFA) 是当前三维定量构效关系(3D-QSAR)研究 的主流方法. 该方法认为当一组同系类药化合物与 生物大分子相互结合时，由于药物分子结构上的差 异而引起分子周围势场分布的改变, 从而直接影响 配基与受体的亲和性. 由于 CoMFA 能够较好反映小 分子药物与生物分子的空间作用情况, 并以直观的 系数等势图的形式给出取代基对生物活性的影响方 式, 因此很快受到了众多相关领域研究者的重视. 随 着CoMFA的成功推广, 包括在环境毒物定量构效关 系的应用 [2], 一系列与之相似的 3D分子性质比较方 法被人们提出, 其代表如比较分子相似性指数分析 (CoMSIA $)^{[3]}$ 、比较分子动量矩分析 (CoMMA $)^{[4]}$ 等. 虽 然CoMFA已在许多具体应用领域取得了较好的效果, 但其仍然存在诸多问题，具体表现在以下几个方面: （1）空间网格划分随意性大; (2) 势能形式不完善; (3) 探针选取标准不确定; (4) 样本构象叠合方式不统一;
(5) 探点位置过于固定. 针对这些缺点当前已经出现 了许多改进方案, 例如Gaillard ${ }^{[5]} 、$ Kellogg ${ }^{[6]} 、$ Waller ${ }^{[7]}$ 等分别将疏水势(MLP)、E-state场、HOMO/LUMO场 引入CoMFA当中; 而DePrist ${ }^{[8]} 、$ Welsh ${ }^{[9]}$ 等则提出了活 性位点匹配规则、基于惯性矩的匹配方法等，这些措 施在一定程度上改善了模型的统计质量和可解释性.

在前人研究的基础上, 本文试图进一步解决 CoMFA 的空间网格划分和探点位置取向问题, 其思 想是通过智能算法优化分子周围探针原子的最佳空 间分布情况, 并在此基础上构建高质量的 3D-QSAR 模型. 为了使用方便这里暂将该法命名为柔性比较 分子场分析法(FCoMFA). FCoMFA 大致通过以下几 步实现: (1) 在叠合好的一组药物分子周围随机产生 一定数目的探针; (2) 以 QSAR 模型的统计量作为评 价函数通过智能算法迭代优化这些探针在空间的位 置取向; (3) 得到探针最优分布模式并给出分子周围 对活性呈正向及负向影响的空间探点图样. 与传统 
的 CoMFA 相比 FCoMFA 有以下几点不同之处: (1) 探 针空间分布并不机械固定于人为划分好的网格之上, 而是由算法自动确定最佳位置, 这样就形成了一种 柔性模型构造机制; (2) 使用较少的探针数量来集中 模拟药物与生物分子(或外界物质)相互作用的活性位 点情况，有效提高了模型的性能; (3) 将不同非键效 应在空间位置上分开处理, 以达到增加模型可解释 性的目的; (4) 避免了药物分子周围某些对模型没有 信息贡献的位点(如结合腔的背面、分子内部等)的引 入而带入噪声和干扰因素.

\section{1 原理和方法}

由于配基与受体相互结合时仅在少数活性位点 发生显著作用，故进行分子场比较过程中找寻药物 分子周围这些位置是决定 FCoMFA 成败的关键. 我 们认为探针空间分布模式比探针数量更为重要, 所 以 FCoMFA 中并不需要过多的探针元素而把重点放 在如何确定它们的具体位置取向.

探针：通常对于具体研究对象而言，由于不同种 类的非键效应对药物的生物活性贡献存在较大差异, 这种差异不仅表现在它们对活性贡献大小上，也表 现在发生非键作用的空间位置上，因此针对不同势 场形式的探针类型应当分别进行考虑. 在 FCoMFA 中对于静电场、立体场和疏水场分别使用 $\mathrm{H}^{+}$离子(质 子)、 $\mathrm{Csp}^{3}$ 原子和 $\mathrm{H}_{2} \mathrm{O}$ 分子作为探针, 表 1 列出了这 些探针的相关参数.

表 1 FCoMFA 中 3 类探针参数

\begin{tabular}{cl}
\hline 探针 & \multicolumn{1}{c}{ 参数 } \\
\hline $\mathrm{H}^{+}$ & 电量: $1.6021892 \times 10^{-19} \mathrm{C}$ \\
$\mathrm{Csp}^{3}$ & van der Waals 半径: $1.70 \times 10^{-10} \mathrm{~m} \frac{[10]}{}$; 势能阱深常数: \\
& $0.309 \mathrm{~kJ} / \mathrm{mol}^{111]}$ \\
$\mathrm{H}_{2} \mathrm{O}$ & 溶剂可及面积: $9.85 \times 10^{-19} \mathrm{~m}^{2}$; 相对疏水 (亲水)常数: $1^{\mathrm{a})}$ \\
\hline
\end{tabular}

a) 由于水分子自身不存在相对疏水(亲水)性, 因此该常数设置为 1

势场：经典药学理论认为药物在抵达受体并与 之发生作用绝大多数都是暂时的、可逆的非键效应, 其表现为静电、立体、疏水、氢键、电荷转移等多方 面因素. 本文考虑到静电、立体、疏水效果几乎包含 了大部分这类信息, 故在 FCoMFA 中主要计算这 3 种作用类型. 对于氢键、电荷转移等可以看成是静电 和立体效应的特殊表现形式.

静电作用(electrostatic interaction)是一类重要的 非键效应，经典的点电荷作用方式服从Coulomb定理，
本文该公式直接来自 CoMFA ${ }^{[1]}$. 由此定义探针 $p$ 与药 物分子的总静电作用能为:

$$
E_{p}(E)=\sum_{i=1}^{n} \frac{1}{4 \pi \varepsilon_{0}} \cdot \frac{q_{p} \cdot q_{i}}{d_{p i}},
$$

其中 $n$ 是药物分子包含的总原子数; $q_{p}$ 和 $q_{i}$ 分别是探 针 $\mathrm{H}^{+}$及药物分子中原子 $i$ 所带电量, 单位为 $\mathrm{C} ; d_{p i}$ 是 探针到原子 $i$ 的 Euclid 距离, 单位为 $\mathrm{m} ; \varepsilon_{0}$ 为真空中的 介电常数 $8.85418782 \times 10^{-12} \mathrm{C}^{2} / \mathrm{J} \cdot \mathrm{m}$.

立体作用(steric interaction)是空间原子间存在的 非偶极-偶极或偶极诱导作用, 这里采用 LennardJones 方程来描述这种作用方式:

$$
E_{p}(S)=\sum_{i=1}^{n} \varepsilon_{p i} \cdot\left[\left(\frac{R_{p i}^{*}}{d_{p i}}\right)^{12}-2 \cdot\left(\frac{R_{p i}^{*}}{d_{p i}}\right)^{6}\right],
$$

上式中 $\varepsilon_{p i}=\left(\varepsilon_{p p} \cdot \varepsilon_{i i}\right)^{1 / 2}$ 为 $\mathrm{Csp}^{3}$ 探针/原子对势能阱深 ${ }^{[11]}$; $R_{p i}{ }^{*}=\left(R_{p}+C_{h} \cdot R_{i}^{*}\right) / 2$, 为经过校正后的探针/受体原子 间van der Waals碰撞半径, 校正因子 $C_{h}$ 由 $\mathrm{Hahn}^{[12]}$ 定义, 当 $i$ 原子 $\mathrm{sp}^{3}$ 杂化时取 $1.00, \mathrm{sp}^{2}$ 杂化取 $0.95, \mathrm{sp}$ 杂化取 0.90; 另外, 各类原子的标准van der Waals半径取自 Bondi 等的报告 ${ }^{[10]}$. 由于Lennard-Jones方程对距离 变化十分敏感, 因此可能在某些靠近化合物原子的 探点处产生非常大的立体作用势能，从而造成QSAR 模型的稳定性变差. 故这里沿用CoMFA的处理方法, 取 $126 \mathrm{~kJ} / \mathrm{mol}$ 作为能量截断值.

疏水作用(hydrophobic interaction)是影响药物分 子与生物体结合的重要因素, 由于其往往表现于体 系熵的改变, 因此很难用一个统一的公式来描述. 对 于有关疏水作用的研究已有众多文献报道 ${ }^{[13]}$, 考虑 到FCoMFA要求深入到药物分子内部原子与 $\mathrm{H}_{2} \mathrm{O}$ 探针 相互作用，同时鉴于Kellogg等提出的hint方法 ${ }^{[14]}$ 已 经成功地用于CoMFA疏水场的计算, 因此我们使用 该法来表达疏水作用势能. 在hint中定义了一个简单 的计算两个原子之间的疏水相互作用表达式:

$$
E_{p}(H)=\sum_{i=1}^{n} S_{p} \cdot a_{p} \cdot S_{i} \cdot a_{i} \cdot e^{-d_{p i}} \cdot T_{p i},
$$

其中 $S$ 为溶剂可及面积(SASA) ${ }^{[15]}$, 是水分子(van der Waals半径为 $1.4 \AA$ )在原子表面滚动其球心形成的表 面面积; $a$ 为相对疏水/亲水性常数, 我们采用Pei等 ${ }^{[16]}$ 定义的原子溶解参数(ASP)作为该值量度; $T$ 是作用形 式的二值判别函数，以表明不同类型原子疏水作用 的熵效应变化方向. 
算法: FCoMFA最终目的是找寻探针在药物分子 周围的最佳分布模式，这直接关系到所建模型的性 能和可解释性, 因此需要一种优良的计算工具来实 现该目标. 近年来智能优化算法的快速发展为此类 问题提供了一个有效的解决途径, 其中又以遗传算 法 (GA) 在药物设计和QSAR研究中最为常见. 但是 FCoMFA并不采用GA进行探针位置优化，而考虑使 用新一代群体智能算法——粒子群优化算法(PSO $)^{[17]}$ 作为计算工具, 这主要有以下几点原因: (1) GA通常 采用 2 进制编码, 对于实数问题则需要进行解码, 这 样会产生计算精度和计算量之间的矛盾，同时还伴 随有诸如Hamming悬崖问题、码串过长、变异可能导 致串变号、串长不易动态变化等缺点. 而PSO则主要 针对连续空间实数求解, 非常适合优化FCoMFA中的 探针坐标; (2) 对于多数问题PSO无论是在种群规模 还是迭代次数上均小于 $\mathrm{GA}$, 因此具有很快的收玫速 度; (3) PSO算法简便、参数较少, 容易在计算机上实 现.

FCoMFA 算法流程如下:

(1) 初始化: 在叠合的药物分子周围分别随机产 生 $n\left(\mathrm{H}^{+}\right), n\left(\mathrm{Csp}^{3}\right)$ 和 $n\left(\mathrm{H}_{2} \mathrm{O}\right)$ 个探针 (通常取 $n\left(\mathrm{H}^{+}\right)=$ $\left.n\left(\mathrm{Csp}^{3}\right)=n\left(\mathrm{H}_{2} \mathrm{O}\right)\right)$, 设 $\left.L=n\left(\mathrm{H}^{+}\right)+n\left(\mathrm{Csp}^{3}\right)+n\left(\mathrm{H}_{2} \mathrm{O}\right)\right)$, 并将所有探针的 $X, Y, Z$ 坐标依次排列形成一个 $3 L$ 维 向量, 该向量就为 PSO 中一个粒子 $i$ 的解空间初始坐 标 $X_{i}=\left(x_{i 1}, x_{i 2}, \cdots x_{i 3 L}\right)^{\mathrm{T}}, i=1,2, \cdots N$. 重复上述过程 $N$ 次, 得到一个包含 $N$ 个粒子的初始种群. 同时每个粒子被 随机赋予一定的飞行速度，该速度同样被表示为一 个 $3 L$ 维向量 $V_{i}=\left(v_{i 1}, v_{i 2}, \cdots v_{i 3 L}\right)^{\mathrm{T}}$.

(2) 适应度计算: PSO 中每一个粒子都对应 L 个 探针在叠合药物分子周围的一种分布情况, 计算出 这些探针与每个药物分子相应的非键作用势能，由 此组成自变量矩阵 $\boldsymbol{X}_{m \times L}(m$ 为样本数, $L$ 为变量数 $)$, 将 其与因变量矩阵 $\boldsymbol{Y}$ (活性)建立偏最小二乘回归(PLS) 模型，并以该模型的留一法交叉检验 $q^{2}$ 作为 PSO 中 粒子的适应度函数.

(3) 叠代过程: PSO 中所有的粒子都有追求自己 所经历过的最优位置 $P_{i}=\left(p_{i 1}, p_{i 2}, \cdots p_{i 3 L}\right)^{\mathrm{T}}$ (“认知”部分) 以及群体经历过的最优位置 $P_{g}=\left(p_{g 1}, p_{g 2}, \cdots p_{g 3 L}\right)^{\mathrm{T}}$ (“社 会”部分)的特性，并通过下式调整第 $i$ 个粒子在第 $d$ 维度上 $(d=1,2, \cdots 3 L)$ 的运动方式:

$$
\begin{aligned}
& v_{i d}^{k+1}=w \cdot v_{i d}^{k}+c_{1} \cdot \operatorname{rand}_{1}^{k} \cdot\left(p_{i d}^{k}-x_{i d}^{k}\right) \\
& +c_{2} \cdot \operatorname{rand}_{2}^{k} \cdot\left(p_{g d}^{k}-x_{i d}^{k}\right), \\
& x_{i d}^{k+1}=x_{i d}^{k}+v_{i d}^{k+1}, \\
& x_{i, k}= \begin{cases}x_{i, k}=X_{\max }, & \text { if } x_{i, k}>X_{\text {max }}, \\
x_{i, k}=X_{\text {min }}, & \text { if } x_{i, k}>X_{\text {min }},\end{cases}
\end{aligned}
$$

$w$ 是惯性权重，用以反映前次速度的影响大小; $c_{1}, c_{2}$ 为学习因子, 分别代表粒子飞向自身最优位置及群 体最优位置的能力; rand 是介于 $[0,1]$ 之间的随机函数; $k$ 为种群迭代次数; $X_{\max }$ 和 $X_{\text {min }}$ 为解空间限制, 通常对 叠合后的分子体系给出 $0.4 \mathrm{~nm}$ 的多余空间.

（4）收敛标准：连续 10 代种群整体的适应度没 有显著改善或到达最大迭代次数 $K$ 则停止计算并输 出结果.

\section{2 结果及分析}

\section{1 糖原磷酸化酶抑制剂}

糖原磷酸化酶 (GP) 是肝糖元分解的限速酶，它 通过将储存在肝脏中的大分子糖元降解为葡萄糖来 为机体提供能量. 采用动物模型实验发现GP的活性 在一定程度上决定着体内的血糖浓度，通过对正常 白鼠的GP进行抑制将导致一系列血糖过低的症状 ${ }^{[18]}$. 上述研究结果表明 $\mathrm{GP}$ 是一个潜在的抗糖尿病药物作 用靶点, 从而引起了人们的广泛兴趣. 47 个葡萄糖类 似物来自文献报道 ${ }^{[19]}$, 它们通过模拟酶底物来竞争 抑制 $\mathrm{GP}$ 的活性，其与 $\mathrm{GP}$ 亲和性大小由结合自由能 $\Delta G$ 表示.

\subsection{FCoMFA 计算}

图 1 给出了 GP 与小分子化合物的复合晶体结构 (PDB ID: 2 GPB), 可以看到在酶活性位点的中心结 合有一分子的 $\alpha-D$-葡萄糖和一分子的磷酸化中间体. 采用处于复合物体系中的 $\alpha-\mathrm{D}$-葡萄糖晶体结构作为 初始药效构象模板(PDB ID: 2GPB), 在 HyperChem 7.0 中手工搭建样本集中其余 46 个分子的立体结构, 并使用 $\mathrm{MM}+$ 力场进行优化(收敛条件设置为 4.18 $\mathrm{J} / \mathrm{mol}$ ) 以消除结构中不合理的部分, 同时利用半经验 量子化学软件 MOPAC 6.0 在 PM3 水平上以单点 (single-point)形式计算出分子中每个原子的净电荷数 目. 将处理完成的分子以*.mol 格式保存并输入到分 子模拟软件 Alchemy 2000 中, 并使用 RMS Fit 模块基 


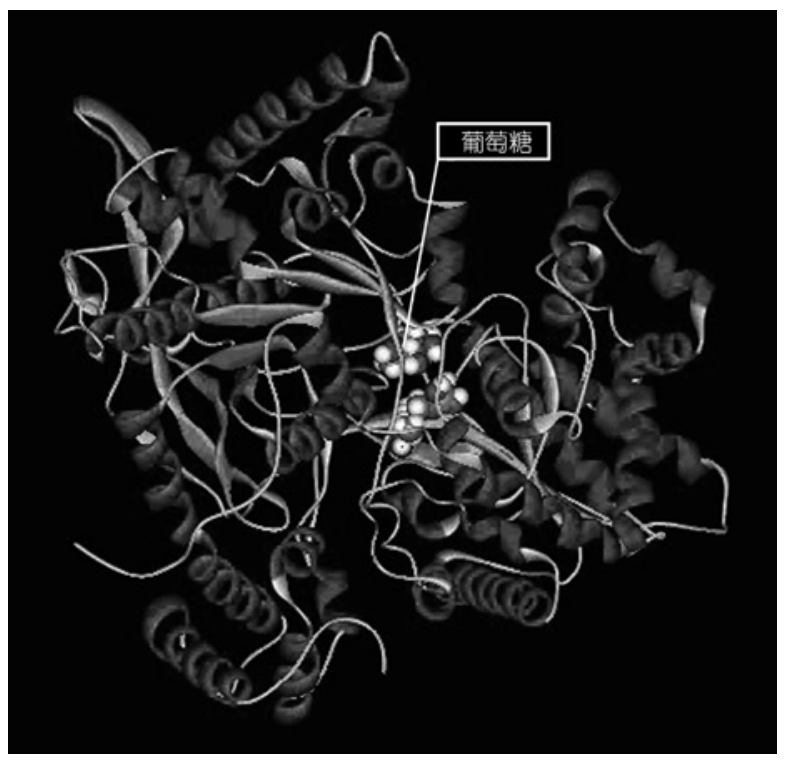

图 1 葡萄糖类似物与 糖原磷酸化酶复合物三维晶体结构

于化合物母环原子进行分子构象叠合.

近年来Tropsha等 [20]研究表明仅凭交叉检验 $q^{2}$ 难 以说明QSAR模型的优劣，模型的预测能力必须通过 外部集进行检验. 鉴于此, 我们从 47 个样本中随机 抽出 7 个分子作为测试集(test set)不直接参与建模, 而用其对训练集(training set)40 个化合物所建模型进 行验证. FCoMFA的参数包括探针数目 $n\left(\mathrm{H}^{+}\right), n\left(\mathrm{Csp}^{3}\right)$ 和 $n\left(\mathrm{H}_{2} \mathrm{O}\right)$ 以及PSO中的惯性权重 $w$ 、学习因子 $c_{1}, c_{2}$ 、 最大迭代次数 $K$. 其中对于惯性权重 $w$ 取 Shi等 ${ }^{[21]}$ 提出 的线性递减策略，即设定该值随迭代过程由初始 0.9 线性递减为 0.4 , 这样有助于算法初期全局搜索性及 后期的局部收敛能力; Kennedy等 ${ }^{[22]}$ 认为学习因子应 当满足 $c_{1}+c_{2} \approx 4$, 推荐为 $c_{1}=c_{2}=2.05$; 另外对于其 他参数设置为 $n\left(\mathrm{H}^{+}\right)=n\left(\mathrm{Csp}^{3}\right)=n\left(\mathrm{H}_{2} \mathrm{O}\right)=10, K=200$. 通过FCoMFA计算得到的结果进一步采用化学计量 学软件Simca-P 10.0 进行深入数据挖掘, 最终获得的 最优PLS模型采用 3 个显著主成分 $(\mathrm{PC})$, 它们包含原 始变量矩阵 $\boldsymbol{X}$ 的 $76.3 \%$ 信息量, 并解释了 $\boldsymbol{Y}$ 变量 $90.8 \%$ 方差, 交叉检验解释 $\boldsymbol{Y}$ 的方差为 $85.1 \%$. 图 2 为训练集 40 个化合物在PLS模型前两个主成分得分空间分布 情况，其中绝大多数样本都落在 $95 \%$ 置信度的 Hotelling $\mathrm{T}^{2}$ 椭圆置信圈内, 仅 24 号和 41 号分子超出 该范围. 经分析发现 24 号化合物为该组样本集中具 有最大结合自由能的分子, 而 41 号化合物的B链则带 有一个 $\mathrm{NH}_{3}{ }^{+}$离子, 由此可以认为这两个分子在得分
图上略微表现特殊是由于自身结构和活性的特殊性 所致. 在图 2 中按样本分子对靶酶亲和性等级进行标 记后发现不同活性的样本有规律的分布在前两个主 成分空间上，活性低的样本主要集中在该图的左下 角，而活性高的则集中在右上方，中等活性的样本分 布在中间，这表明PLS模型主成分空间能够较好反映 分子周围非键场能与亲和性之间的内在联系。进一 步使用上述模型对 7 个测试集化合物进行预测，从图 3 中我们可以直观地看出预测值与实验值非常接近, 所得结果的复相关系数 $r^{2}$ pred 及均方根误差RMSEP分 别为 0.679 和 0.767 , 由此证实了 $\mathrm{FCoMFA}$

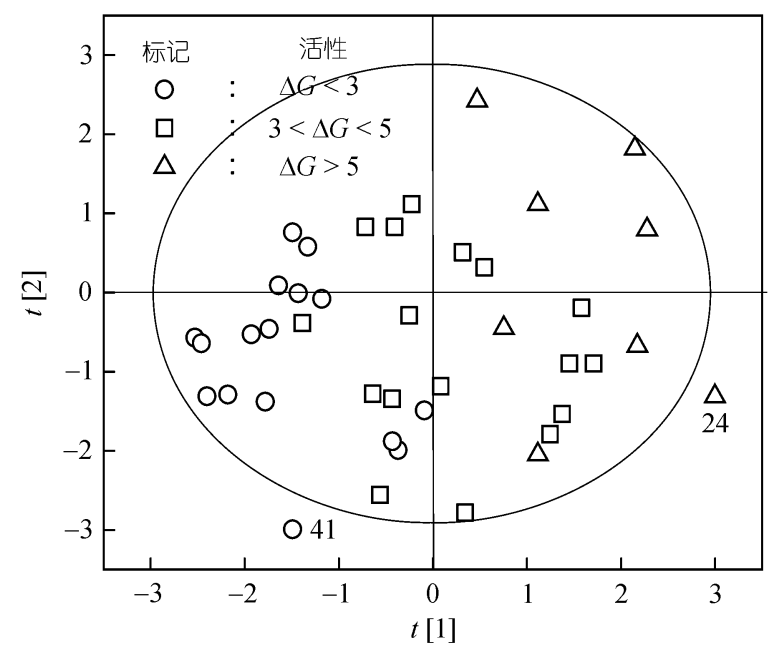

图 2 PLS 模型中 40 个训练集样本在前两个 主成分 $(t[1] / t[2])$ 上的得分分布散点图

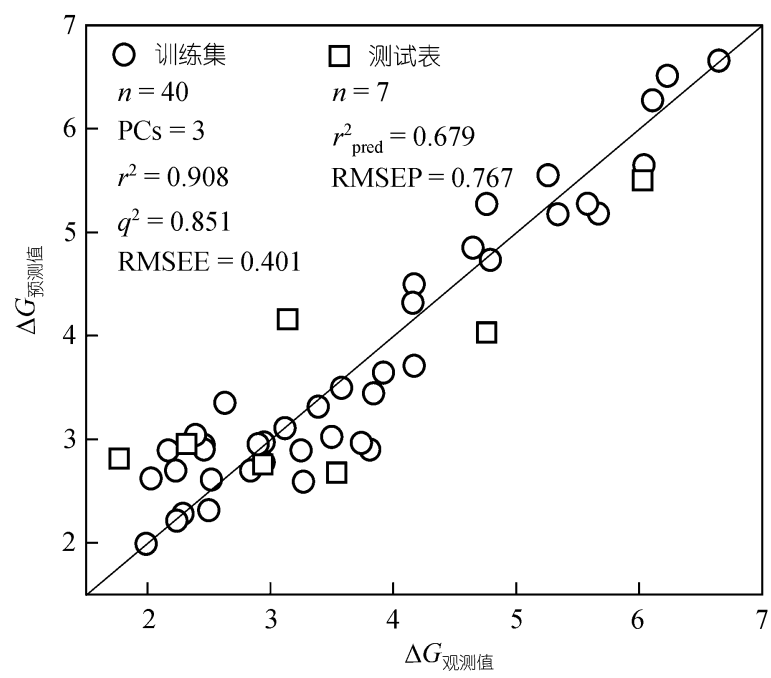

图 347 个糖原磷酸化酶抑制剂与受体结合自由能的 模型计算值与实验观测值的相关情况 

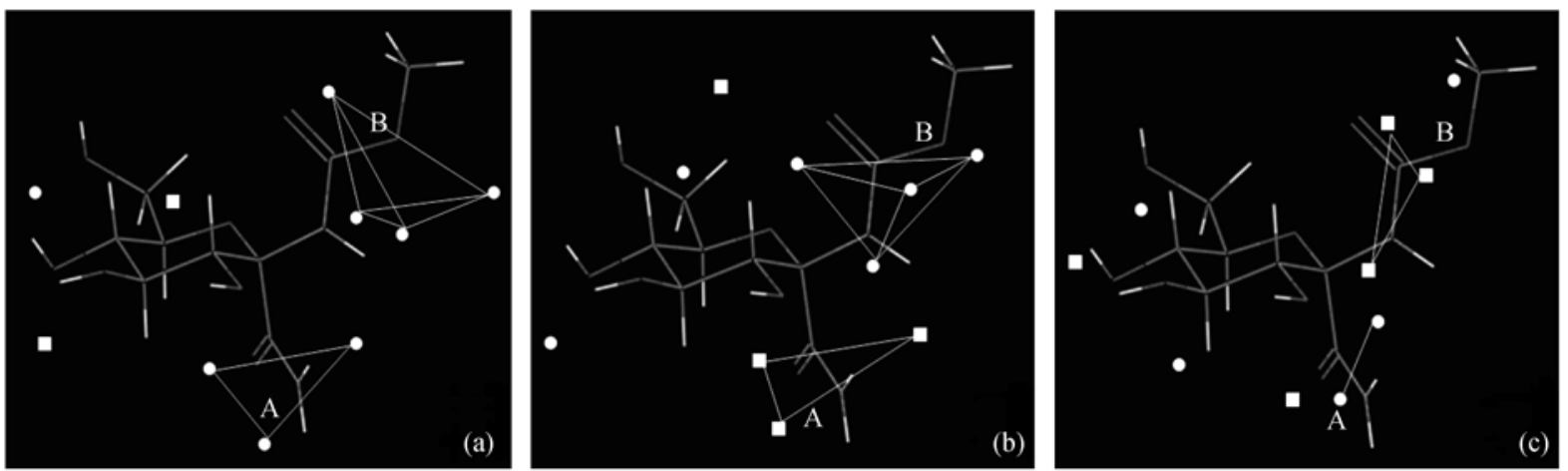

图 4 FCoMFA 探针分布模式图

(a) 静电探针; (b) 立体探针; (c) 疏水探针

所建模型具有较强的稳定性和泛化能力.

\section{3 探针分布模式图}

图 4(a) ( (c)分别是 FCoMFA 计算得到的静电、 立体、疏水探针在活性最高样本周围空间分布情况, 其中不同形状代表通过对 PLS 模型似然方程系数分 析得到的探针所处位置分子势场对活性正反向贡献 情况. 由于本文所使用的 47 个糖原磷酸化酶抑制剂 的结构差异仅表现在母环 1 位侧链上, 为了便于观察 我们将图中处于此处对活性具有相同贡献的探针连 接起来. 从图 4(a)可以看到分子周围分布着较多增加 负电势有利提高分子亲和性的探针(圆形，下文简称 负电势探针), 特别是 $\mathrm{B}$ 链周围以此类探针为主. 经 分析发现多数对靶酶具有高亲和性的样本其 B 链往 往含有较多电负性较大的杂原子, 如 $\mathrm{O}, \mathrm{N}, \mathrm{Cl}, \mathrm{Br}$ 等, 从而导致此处电势显著下降. 而 $\mathrm{A}$ 链周围正(方形)/ 负电势探针皆有存在, 说明该链电性对药物分子的 亲和性影响较小; 进一步观察图 4(b)立体探针分布可 知 $\mathrm{B}$ 链周围存在大量圆形探针指示该处存在大的取 代基有利于配基亲和性的增加，而 $\mathrm{A}$ 链周围方形探 针则表明该侧链情况与 $\mathrm{B}$ 链正好相反, 需小体积取代 基为佳. 该结论高亲和性 $(\Delta G>20.90 \mathrm{~kJ} / \mathrm{mol})$ 化合物 情况符合较好, 这些分子的 $\mathrm{B}$ 链都具有一个较大取代 基而 $\mathrm{A}$ 链则为一个很小的氢原子; 图 4(c)的疏水探针 分布情况没有上述静电和立体探针的规律性强, 在 A, B 链周围都存在两种形状的探针说明疏水性对糖 原磷酸化酶抑制剂的亲和性影响较小, 不是驱动配 基与受体结合的主要因素, 该结论得到了 PLS 变量 载荷值的支持 $(8 / 10$ 的疏水探针对第一个主成分贡献 loading $<0.3$ ). 其中 B 链周围 3 个方形探针表明该处
有利于亲水性取代基存在，这可能是由于负电性取 代基往往带有较多杂原子，从而间接影响该基团周 围疏水分布的缘故. 另外, 我们从 3 幅图都可以看到 在远离 $\mathrm{A}, \mathrm{B}$ 链处仍然存在少量探针, 这些探针分布 规律性不强, 可以认为是理论模型与实际情况偏差 所致.

\section{3 结语}

CoMFA 作为一种经典的 3D-QSAR 研究方法至 提出以来已被众多相关领域科学工作者所采用, 并 且至今仍然是药物设计的主流研究工具. 但是 CoMFA 仍然存在诸多缺点, 这主要体现在其探针选 取、势场形式、叠合方案、网格划分等方面, 纵观当 前对 CoMFA 的改进主要是从前三点来考虑, 而对于 直接影响建模结果的最后一点即网格划分却研究较 少. 鉴此, 于本文中取消了传统 CoMFA 中作为探针 安置的网格格点, 并辅以 PSO 算法来优化不同类型 探针在药物分子周围最佳分布模式, 以此把柔性机 制引入到 CoMFA 当中, 从而形成了一种新的 3D-QSAR 研究方法: 柔性比较分子场分析法 (FCoMFA). 文中使用 FCoMFA 对 47 个糖原磷酸化酶 抑制剂进行了系统的 QSAR 研究, 结果表明 FCoMFA 所建模型具有较高的稳定性及预测能力, 所给出的 探针分布模式图正确地反映了不同取代基团对该组 样本亲和性的影响效果. 这些结论对糖原磷酸化酶 抑制剂活性笁选和结构改造具有一定的指导意义. 另外, 由于本文中取消了传统分子场中探针安置网 格点, 所形成的新 3D-QSAR 柔性比较分子场分析法 (FCoMFA)方法具有一定普适性, 可望象比较分子场 分析法(CoMFA)一样推广应用到医农药物和环境毒 
物定量构效关系研究等方面.

致谢 感谢化学生物学与分子药物学研究室田菲菲 博士生、杨善涁副教授、吴世容、兰玉坤工程师等，药 物设计学和环境毒理学研究室梅虎博士、周原副教 授、刘树深教授、杨胜喜、张梦军讲师、李根容、覃 仁辉工程师、张巧霞硕士等提供有关帮助或协助, 谨 致谢意.

\section{参考文献}

1 Cramer R D, Patterson D E, Bunce J D. Comparative molecular field analysis (CoMFA). 1. Effect of shape on binding of steroids to carrier proteins. J Am Chem Soc, 1988, 110: 5959-5967[DOI]

2 王晓栋, 林志芬, 尹大强, 刘树深, 王连生. 硝基芳烃致突变性 的二维/三维 QSAR 比较研究. 中国科学 B 辑: 化学, 2004, 34(6): $498-503$

3 Silverman B D, Platt D E. Comparative molecular moment analysis (CoMMA): 3D-QSAR without molecular superposition. J Med Chem, 1996, 39: 2129-2140[DOI]

4 周鹏, 全建波, 田菲菲, 等. 一种新的比较分子/虚拟受体相互 作用分析法. 科学通报, 2006, 51(12): 1390-1394

5 Gaillard P, Carrupt P-A, Testa B, Schambel P. Binding of arylpiperazines, (aryloxy) propanolamines, and tetrahydropyridylindoles to the 5- $\mathrm{HT}_{1 \mathrm{~A}}$ receptor: contribution of the molecular lipophilicity potential to three-dimensional quantitative structure-affinity relationship models. J Med Chem, 1996, 39(1): 126$134[\mathrm{DOI}]$

6 Kellogg G E, Kier L B, Gaillard P, Hall L H. The E-state fields. Application to 3D QSAR. J Comp -Aid Mol Des, 1996,10: 513$520[\mathrm{DOI}]$

7 Waller C L, Marshall G R. Three-dimensional quantitative structure-activity relationship of angiotesin-converting enzyme and thermolysin inhibitors. II . A comparison of CoMFA models incorporating molecular orbital fields and desolvation free energies based on active-analog and complementary-receptor-field alignment rules. J Med Chem, 1993, 36: 2390-2403[DOI]

8 DePriest S A, Mayer D, Naylor C B, Marshall G R. 3D-QSAR of angiotensin-converting enzyme and thermolysin inhibitors: a comparison of CoMFA models based on deduced and experimentally determined active site geometries. J Am Chem Soc, 1993, 115(13): 5372-5384[DOI]

9 Welsh W J, Tong W D, Collantes E R, Chickos J S, Gagarin S G.
Enthalpies of sublimation and formation of polycyclic aromatic hydrocarbons (PAHs) derived from comparative molecular field analysis (CoMFA) - application of moment of inertia for molecular alignment. Thermochem Acta, 1997, 290: 55-64[DOI]

10 Bondi A. van der Waals volumes and radii. J Phys Chem, 1964, 68(3): $441-451$

11 Levitt M. Protein folding by restrained energy minimization and molecular dynamics. J Mol Biol, 1983, 170: 723-764[DOI]

12 Hahn M. Receptor surface models. 1. Definition and construction. J Med Chem, 1995, 38(12): 2080-2090[DOI]

13 Tanford C. The hydrophobic effect and the organization of living matter. Science, 1978, 200: 1012-1018

14 Kellogg G E, Semus S F, Abraham D J. HINT - a new method of empirical hydrophobic field calculation for CoMFA. J ComputAided Mol Des, 1991, 5: 545-552[DOI]

15 Hasel W, Hendrikson T F, Still W C. A rapid approximation to the solvent accessible surface areas of atoms. Tetrahed Comp Method, 1988, 1: 103-116[DOI]

16 Pei J, Wang Q, Zhou J, Lai L. Estimating protein-ligand binding free energy: atomic solvation parameters for partition coefficient and solvation free energy calculation. Proteins, 2004, 57: 651$664[\mathrm{DOI}]$

17 Kennedy J, Eberhart R C. Particle swarm optimization. Proceedings of IEEE International Conference on Neut ral Networks. Perth: IEEE Service Center, 1995. 1942-1948

18 Bollen M, Hue L, Stalmans W. Effects of glucose on phosphorylase and glycogen synthase in hepatocytes from diabetic rats. Biochem J, 1983, 210: 783-787

19 Venkatarangan P, Hopfinger A J. Prediction of ligand-receptor binding free energy by $4 \mathrm{D}-\mathrm{QSAR}$ analysis: application to a set of glucose analogue inhibitors of glycogen phosphorylase. J Chem Inf Comput Sci, 1999, 39: 1141-1150[DOI]

20 Tropsha A, Gramatica P, Gombar V K. The importance of being earnest: validation is the absolute essential for successful application and inerpretation of QSPR models. QSAR Comb Sci, 2003, 22: 69-77[DOI]

21 Shi Y, Eberhart R C. Empirical study of particle swarm optimization. Proceedings of the IEEE International Conference on Evolutionary Computation Piscataway: IEEE Service Center, 1999: $1945-1950$

22 Kennedy J. Thinking is social: experiments with the adaptive culture model. J Conf Res, 1998, 42: 56-76 